\title{
Reabilitação da hemiparesia pós-acidente vascular encefálico com mirror visual feedback Hemiparesia rehabilitation post-cerebrovascular accident through mirror visual feedback
}

Claudia Morais Trevisan, Ft.,M.Sc.*, Carlos Bolli Mota, D.Sc.**, Rosana Marin de Oliveira, Esp.**, Maria Elaine Trevisan**, Ft.,M.Sc., Riccardo Pratesi, D.Sc. ${ }^{* * *}$

*Pós-graduação da Faculdade de Ciências da Saúde, Universidade de Brasilia, ${ }^{* *}$ Laboratório de Biomecânica, Centro de Educação Física e Desportos, Universidade Federal de Santa Maria, ${ }^{* *}$ Departamento de Fisioterapia, Centro de Ciências da Saúde, Universidade de Brasilia, ${ }^{* * *}$ Departamento de Fisioterapia, Centro de Ciências da Saúde, Universidade Federal de Santa Maria, ${ }^{* * * * o o ́ s-}$ graduação da Faculdade de Ciências da Saúde, Universidade de Brasilia

\section{Resumo}

O objetivo deste estudo piloto foi investigar a efetividade do método de reabilitação baseado no mirror visual feedback em paciente apresentando precário uso funcional do membro superior devido comprometimento motor e principalmente sensorial, pós-acidente vascular encefálico, em fase crônica. Foi realizada avaliação cinemática do movimento de membro superior, antes e após quatro semanas de mirror visual feedback, quantificando ângulos de movimentação articular e tempos, em tarefas de alcance e preensão, utilizando-se o sistema automatizado de vídeo Peak Performance ${ }^{T M}$. Nos resultados apresentados no pós-teste, tanto as curvas representativas do movimento como o tempo de execuçấo das tarefas do lado afetado aproximaram-se sensivelmente às respostas observadas no membro não-afetado. $\mathrm{O}$ presente estudo sugere que a reabilitação pelo mirror visual feedback é um método efetivo na recuperaçấo de membro parético pós-acidente vascular encefálico, mesmo em fase crônica.

Palavras-chave: biomecânica, acidente vascular encefálico,

hemiplegia, reabilitação.

\begin{abstract}
The objective of this pilot study was to investigate the effectiveness of a rehabilitation method based on mirror visual feedback in a patient with precarious upper limb function due to contralateral motor, and mainly sensorial, deficits caused by a stroke. It was carried out a kinematic evaluation of the upper limb movement, four weeks before and after using the mirror visual feedback technique, quantifying articular movement angles and times, in reaching and grasping tasks, using the automatic Peak Performance ${ }^{T M}$ video system. The results showed in the post test that both curves representing movement and task execution time of the affected side are nearly similar to the non-affected limb. The present study suggests that rehabilitation with mirror visual feedback is an effective method for recovering paretic upper limb postcerebrovascular accident, even in a chronic stage.
\end{abstract}

Key-words: biomechanics, stroke, hemiplegia, rehabilitation.

\section{I ntrodução}

Indivíduos com hemiplegia pós-acidente vascular encefálico (AVE) demonstram espasticidade, fraqueza muscular e deficiência permanente na coordenação do movimento [1]. A recuperação neurológica e funcional desses pacientes ocorreria principalmente dentro dos cinco a seis primeiros meses, acreditando-se não haver significativo progresso após este prazo [2]. No entanto, atuais conceitos concernentes à plasticidade do cérebro sugerem que, mesmo após prazos prolongados, conexôes corticais podem ser remodeladas por estímulos sensoriais levando a modificaçóes das áreas de representação cortical, com conseqüente melhora da capacidade motora do membro parético $[3,4]$.

Estudos recentes têm evidenciado que métodos que integrem estímulos sensoriais à resposta motora são efetivos na recuperação do comprometimento motor pós-AVE [5]. A reaprendizagem motora em pacientes com seqüela de AVE [6] neste enfoque baseou-se em estudos efetuados por Ramachandran $e t$ al. em pacientes com síndrome do membro fantasma [7-10]. As 
sensaçóes anômalas e freqüentemente dolorosas percebidas por estes pacientes podiam ser revertidas e substituídas por vívidas sensações sinestésicas do membro fantasma ao serem observados os movimentos do membro sadio em espelho, técnica denominada mirror visual feedback (MVF) [10]. O mecanismo subjacente a este processo, a hipótese de "remapeamento" de Ramanchandran et al. $[8,9]$ não está ainda completamente esclarecido, mas seria possivelmente conseqüente a estímulos sensoriais que invadiriam e ativariam zonas adjacentes às áreas desaferentadas no córtex e cerebelo. O uso de estímulos de um sistema sensorial intacto, a visão, no recrutamento de circuitos neuronais silenciosos permitiu propor uma abordagem completamente nova em reabilitação neurológica.

\section{Relato de caso}

$\mathrm{O}$ primeiro paciente admitido nos critérios de inclusão foi do sexo masculino, 42 anos, com história de hemiplegia de início súbito, ocorrida 16 meses antes da pesquisa, com comprometimento dimidiado, à esquerda, predominante em um dos membros superiores. A tomografia computadorizada mostrou hematoma intra-parenquimatoso temporoparietal à $\mathrm{D}$, comprometendo inclusive o tálamo, com edema adjacente. Apresentou leve disartria, ausência de comprometimento cognitivo, déficits cognitivos e história de lesões prévias. Referiu considerável dificuldade em utilizar seu lado esquerdo na ausência da visão, na apreensão de objetos e na localização espacial no lado afetado. Encontramos ainda escore de espasticidade $1^{+}$na escala de Ashworth [11] com leve aumento no tônus muscular, mais pronunciado distalmente (força de preensão manual grau 3), zero na escala analógica da dor, moderada hiperreflexia, pronunciado tremor intencional e dismetria na prova index-nariz. Em sua história médica, encontramos hipertensão arterial subcontrolada e abuso de álcool. O protocolo da pesquisa foi aprovado pelo Comitê de Ética em Pesquisa da Universidade Federal de Santa Maria.

A técnica de MVF foi aplicada com o paciente ergonomicamente sentado em frente ao espelho de 45 por $60 \mathrm{~cm}$ perpendicularmente posicionado em frente ao seu tórax. Foram realizadas três sessóes semanais domiciliares de trinta minutos de duração durante um período de quatro semanas.

Figura 1-Paciente executando a técnica MVF.

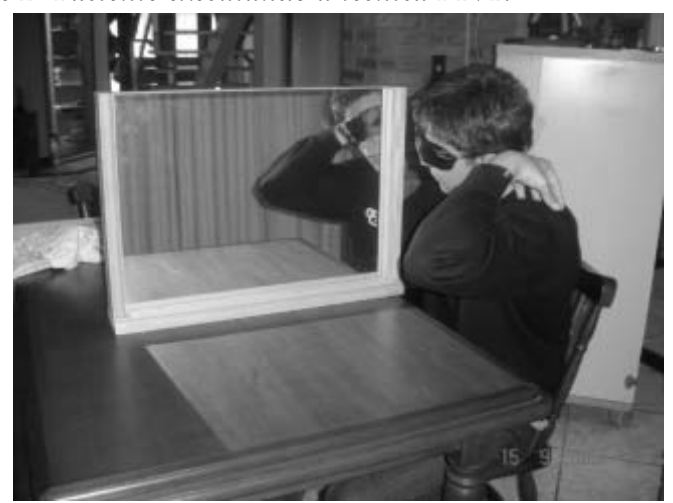

Os movimentos do MS foram avaliados, pré e pós-reabilitação, em tarefas de alcance e preensão [12] (Tarefa 1 - alcançar o alvo situado sobre a mesa; Tarefa 2 - alcançar o alvo e levar a máo à boca; Tarefa 3 - levar a máo acima da cabeça) por avaliação cinemática realizada pelo sistema Peak Performance ${ }^{T M}$ (Peak Performance Technologies, Inc Colorado, USA). As modificaçôes seqüenciais nos ângulos de movimento articular e o tempo de realização das tarefas foram analisados de forma quantitativa nos planos sagital, frontal e transverso.

No presente estudo, a avaliação cinemática na fase pré e pós-reabilitação foi exemplificada pelas modificações dos ângulos dos movimentos do ombro afetado e náo-afetado somente pela tarefa 1 (alcance do alvo situado sobre a mesa e retorno). A análise dos dados obtidos foi realizada utilizando o software IDL versão 6.3, sendo as imagens capturadas equiparadas em 500 quadros, permitindo a comparação dos resultados do pré e pós-teste do lado afetado com o não-afetado, independendo do tempo de realização da tarefa.

Como pode ser observado na figura 2, a curva representativa do ombro afetado, no pré-teste, apresentou maior variaçáo dos movimentos articulares, resultando em traçado mais irregular, traduzindo uma expressiva incoordenação na execução do movimento. Por outro lado, a curva representativa do ombro afetado, no pós-teste, revelou-se mais uniforme, aproximando-se sensivelmente a do lado não-afetado, com ausência de variaçôes significativas na amplitude do movimento, traduzindo a melhora obtida na coordenação motora.

Figura 2 - Variação angular no ciclo de eventos do ombro afetado e não afetado, na execução da tarefa 1, pré e pós-reabilitação, pósequiparação dos resultados pelo IDL.

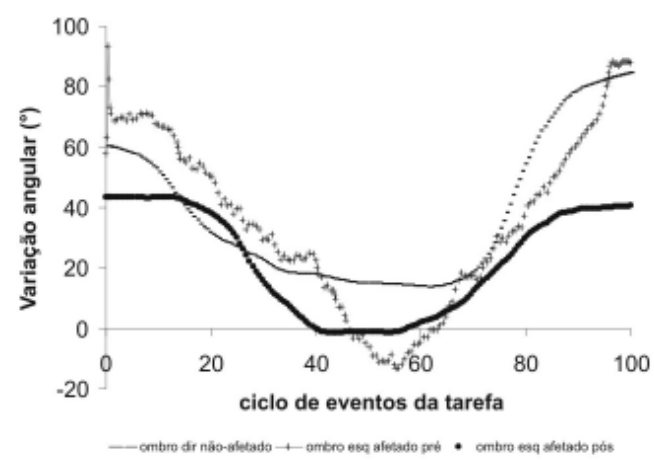

O tempo de execução das tarefas programadas também revelou nítida melhora. Após utilização da MVF, a análise demonstrou expressiva redução no tempo de execução no membro afetado. Comparando-se os resultados obtidos no pré e no pós- teste foi observado um decréscimo de $81 \%$ na execução da tarefa 1 , de $77,3 \%$ na tarefa 2 e de $68,5 \%$ na tarefa 3. 


\begin{tabular}{llll}
\multicolumn{2}{l}{ Tabela I - Variação do tempo (em segundos) } & pré e pós $M V F$ \\
\hline Tarefa & $\begin{array}{l}\text { Lado afetado } \\
\text { Pré-teste }\end{array}$ & $\begin{array}{l}\text { Lado não-afetado } \\
\text { Pós-teste }\end{array}$ \\
\hline T1 & $32,25 \mathrm{~s}$ & $6,12 \mathrm{~s}$ & $3,47 \mathrm{~s}$ \\
T2 & $39,58 \mathrm{~s}$ & $9 \mathrm{~s}$ & $4,73 \mathrm{~s}$ \\
T3 & $35,92 \mathrm{~s}$ & $11,3 \mathrm{~s}$ & $5,73 \mathrm{~s}$ \\
\hline
\end{tabular}

(T1 - alcance do alvo situado sobre a mesa, T2- alcance do alvo após levar a mão à boca e T3 - levar a mão acima da cabeça)

\section{Discussão}

A nítida melhora obtida na função motora pela aplicação da técnica de MVF foi objetivamente evidenciada pela avaliação cinemática dos movimentos do membro afetado antes e depois da terapia. Este paciente que vinha sendo submetido a métodos convencionais de reabilitaçáo durante os prévios 16 meses, com mínimos resultados práticos, apresentou apreciável recuperação funcional com boa melhora da coordenaçáo motora.

É interessante observar que a impressionante recuperação funcional deste paciente no breve espaço de quatro semanas ocorreu apesar do profundo comprometimento sensorial. Esta observação é coincidente com o caso descrito por Sathian et al. [13] em que também houve uma expressiva melhora funcional ao estímulo visual ilusório fornecido pelo espelho, sem paralela recuperação da sensibilidade.

Os mecanismos subjacentes à eficiência da MVF na recuperação funcional de membros paréticos não estão completamente esclarecidos, mas são provavelmente dependentes da plasticidade neural demonstrada por remapeamento topográfico que já foi evidenciado em diversos estudos [14,15]. Áreas cruciais na recuperação funcional neste e provavelmente em outros pacientes englobam aquelas que apresentam tanto representaçôes sensoriais multi-modais quanto uma contribuição para o controle motor. A existência destas áreas foi demonstrada em primatas, incluindo o homem, e se situam no córtex parietal e frontal [10].

É fato conhecido que pacientes com perda sensorial apresentam uma maior dificuldade de reabilitação funcional e as técnicas usualmente utilizadas enfocam predominantemente a recuperação da função motora. Entretanto, os pacientes com alteraçôes somatossensoriais podem se constituir no grupo mais adequado à MVF exatamente devido a sua dependência do estímulo visual. Isto é coincidente com progressos observados em nossos pacientes, ainda em estudo submetidos à mesma técnica, nos quais constatamos resposta à MVF mais lenta naqueles sem comprometimento sensorial apreciável.

\section{Conclusão}

Este estudo piloto confirma a eficácia do método de reabilitação baseado na técnica MVF em paciente pós-AVE em fase crônica com comprometimento predominantemente sensorial. No entanto, outros estudos tornam-se necessários, com maior número de pacientes, para reforçar a efetividade deste método e determinar se uma resposta consistente pode ser obtida mesmo em pacientes com comprometimento predominantemente motor.

\section{Referências}

1. Stevens JA, Stoykov MEP. Using motor imagery in the rehabilitation of hemiparesis. Arch Phys Med Rehabil 2003;84:1090-2.

2. Jørgensen HS, Hirofumi N, Raaschou HO, Jørgen VL, Støier $\mathrm{M}$, Olsen T. Outcome and time course of recovery in stroke. Part II: Time course of recovery. The Copenhagen study. Arch Phys Med Rehabil 1995;76:406-12.

3. Chen R, Cohen LG, Hallett M. Nervous system reorganization following injury. Neuroscience 2002;111:761-73.

4. Page $S$. Reconsidering the motor recovery plateau in stroke rehabilitation. Arch Phys Med Rehabil 2004;85:1377-80.

5. Barreca S, Wolf SL, Fasoli S, Bohanon R. Treatment interventions for paretic upper limb of stroke survivors: a critical review. Neurorehabil Neural Repair 2003;17(4):220-6.

6. Altschuler EL, Wisdom SB, Stone L, Foster C, Galasko D, Llewellyn ME, et al. Rehabilitation of hemiparesis after stroke with a mirror. Lancet 1999;353:2035-6.

7. Ramachandran VS. Behavioral and magnetoencephalographic correlates of plasticity in the adult human brain. Proc Nat Acad Sci USA 1993;90:10413-20.

8. Ramachadran VS, Rogers-Ramachadran D, Cobb S. Touching the phantom limb. Nature 1995;377:489-90.

9. Ramanchandran VS, Rogers-Ramanchandran D. Phantom limbs and neural plasticity. Arch Neurol 2000;57:317-20.

10. Ramanchandran VS. Plasticity and functional recovery in neurology. Clin Med 2005;5:368-73.

11. Bohannon RW, Smith MB. Interrater reliability of a modified Ashworth scale of muscle spasticity. Phys Ther 1987;67(2):2067.

12. Lyle RC. A performance test for assessment of upper limb function in physical rehabilitation treatment and research. Int J Rehab Research 1981;4:483-99.

13. Sathian K, Greenspan AL, Wolf Sl. Doing with mirror: a case study of a novel approach for rehabilitation. Neurorehabil Neuro Repair 2000;14(1):73-6.

14. Nudo RJ, Wise BM, SiFuentes F, Milliken GW. Reorganization of movement representations in primary motor cortex following focal ischemic infarcts in adult squirrel monkeys. J. Neurophysiology 1996;75(5):2144-9.

15. Pascual-Leone A, Torres F. Plasticity of the sensorimotor cortex representation of the reading finger in Braille readers. Brain 1993;119:1849-57. 